\title{
Imaging of Three-Dimensional Epithelial Architecture and Function in Cultured CaCO2A Monolayers with Two-Photon Excitation Microscopy
}

\author{
RICHARD J. GILBERT,* MATTHEW HOFFMAN, ADAM CAPITANO, AND PETER T.C. SO \\ Department of Mechanical Engineering, Massachusetts Institute of Technology; Cambridge, Massachusetts 02139
}

\author{
KEY WORDS two-photon excitation microscopy; CaCo2A epithelial cell monolayers; gastroin- \\ testinal tract
}

\begin{abstract}
The principal functions of the gastrointestinal tract mucosa include nutrient absorption, protein and fluid secretion, and the regulated symbiosis with intraluminal contents. Research in epithelial biology has benefited significantly from the use of cultured monolayer preparations, which closely replicate the structure and function of normal gastrointestinal mucosa. Given the explicit importance of epithelial architecture to its physiology, investigations of epithelial biology should be enhanced by the capacity to track microscopic structures and substances in live cells. In order to achieve this goal, it is necessary to employ a microscopic technique with the capability of imaging deep into the tissue or cell preparation, without adversely affecting its physiology. Two-photon excitation microscopy may constitute such a technique, due to its ability to provide fluorescence excitation of fluorophores using near infrared radiation, that has lower tissue absorption and scattering coefficients. This allows the efficient collection of light energy from sites hundreds of microns deep, with only minimal tissue damage. In this report, we have presented an introduction to the theory and practice of two-photon microscopy for imaging the GI tract epithelium, and have presented examples of its utility in discerning three-dimensional structure and function in CaCo2A epithelial cell monolayers. Microsc. Res. Tech. 51:204-210, 2000. ๑ 2000 Wiley-Liss, Inc.
\end{abstract}

\section{INTRODUCTION}

Novel insights into the biology of the gastrointestinal tract have emerged in recent years through the use of culture monolayer preparations of gut epithelium (Madara, 1990). The ability to simulate the morphology and function of the gut mucosa in a cell culture environment has been particularly useful for discerning the basis of such functions as nutrient absorption (Perdikis and Basson, 1997) and signal transduction (Nusrat et al., 1995), and for elucidating the pathological mechanisms of infectious and inflammatory diseases of the GI tract (Hurley et al., 1999; McCormick et al., 1993; Michalsky et al., 1997; Wick et al., 1991).

Mature epithelial cells grown in culture configure themselves into monolayer formations, whose constituting cells possess polarized, crypt-like, columnar structure, and demonstrate high electrical resistance (Madara, 1998; Madara et al., 1987; Quaroni and Beaulieu, 1997). These cells also exhibit a well-differentiated brush border on the apical surface (Peterson and Mooseker, 1993), and express characteristic small intestinal microvillus transporters of nutrients, pathogens, and inflammatory mediators. Significantly, these culture monolayers are dynamic and tightly regulated, analogous to their behavior in vivo, and thus comprise excellent models to study normal and pathological function. Nonetheless, current methods of studying their biology suffer from the basic limitation that they are unable to image a microscopic structure in live cells or tissue, without adversely affecting its function.
Two-photon excitation microscopy, a novel microscopic imaging approach, constitutes a method for simultaneously visualizing in situ architecture and function in epithelial tissue. This is related to its ability to scan a given cell or tissue with an excitation pulsation configured to excite fluorophores, based on the absorption of two (or more) photons at discrete locations. The reduced scattering and absorption of infrared excitation light thus allows the collection of data from sites deep into the specimen with minimal photo-damage (Centonze and White, 1998; Denk et al., 1990; Masters et al., 1997). In this report, we have described the application of two-photon excitation microscopy for the imaging of the GI tract epithelium. We have provided a primer regarding two-photon theory and methodology, and have presented evidence of its capability to resolve $3 \mathrm{D}$ structure and function in a cultured epithelial cell line, $\mathrm{CaCo} 2 \mathrm{~A}$ cells.

\section{MATERIALS AND METHODS Cell Culture}

CaCo2A cells were perpetuated from frozen stocks in $\mathrm{T}_{75}$ flasks incubated at $37^{\circ} \mathrm{C}, 5 \% \mathrm{CO}_{2}$, and fed with $15 \mathrm{~mL} /$ flask Dulbecco's modified eagle medium (DMEM), supplemented every 48 hours with high glu-

*Correspondence to: Richard J. Gilbert, M.D., Department of Mechanical Engineering, Massachusetts Institute of Technology, 77 Massachusetts Avenue, Cambridge, MA 02139. E-mail: rgilbert@mit.edu

Received 20 June 2000; accepted in revised form 5 July 2000 
Fig. 1. Principals of two-photon excitation. Efficient two-photon excitation is created when the density of photons per unit time and unit volume becomes high enough for two photons to be absorbed by the same chromophore. The absorbed energy for that chromophore is the sum of the energies of the two photons. Left: In two-photon microscopy, the conversion from the ground state to the excited state occurs only when both photons are absorbed simultaneously, an event of relatively low probability. Right: In two-photon excitation, only the fluorescence constituted in a highly discrete excitation volume is excited. The systematic variation of this focal point through all spatial and temporal domains creates a complete $3 \mathrm{D}$ image per unit time. (Note color version of artwork is available online.)
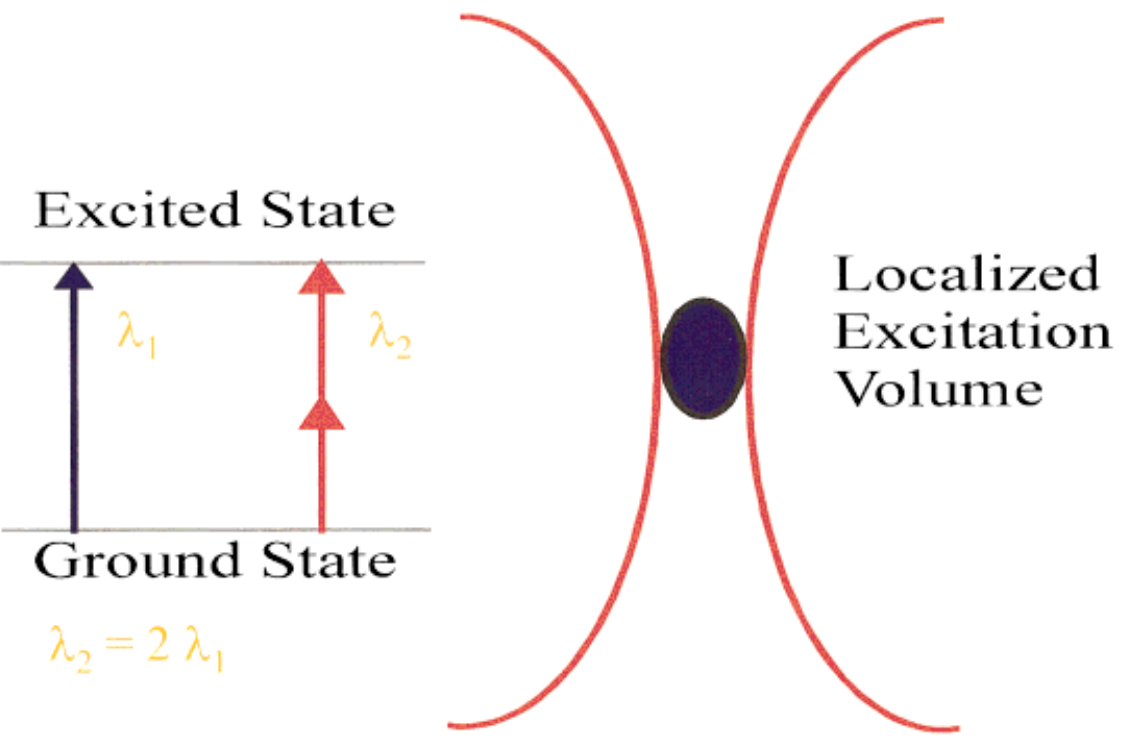

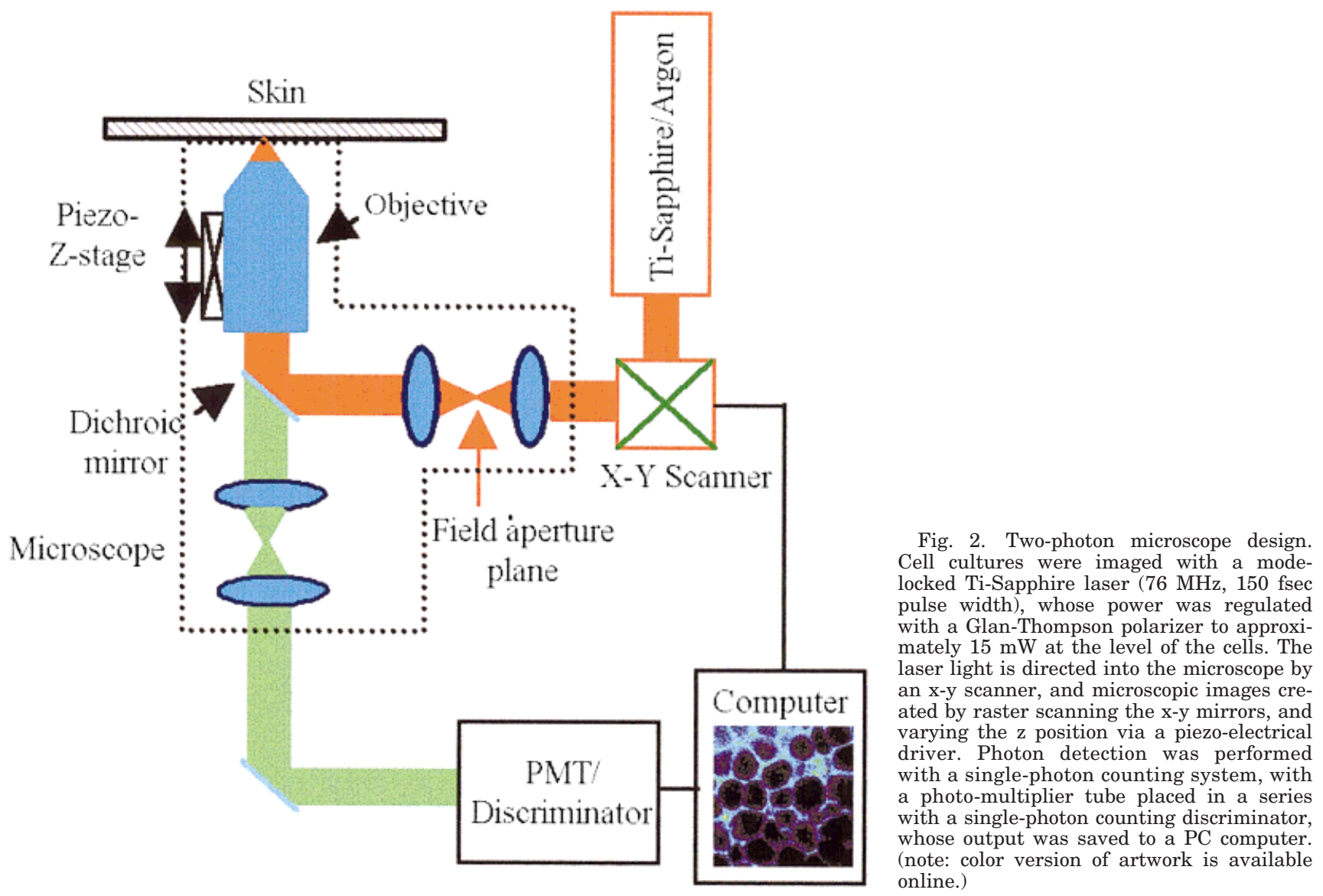

cose, $10 \%$ fetal calf serum, $1 \times$ phosphate buffered saline, and $25 \mathrm{mM}$ HEPES. Upon reaching $90 \%$ confluence, the cells were washed with Dulbecco's phosphate buffered saline, incubated with $1 \mathrm{~mL}$ T-EDTA, diluted to $\frac{1}{10}$ of the pre-passaged monolayer, and
$100 \mathrm{ml}$ of this cell suspension was added to $6.5-\mathrm{mm}$ polystyrene Transwell filter membranes (Costar). These membranes were previously coated with $1 \mathrm{ml}$ purified rat tail collagen, and suspended in $100 \mathrm{ml}$ of $60 \%$ ethanol. Within 7-10 days, the cells grew to con- 
fluence. At confluence, the maturity of the monolayer was assessed as a function of its electrical resistance, with those monolayers possessing a resistance $>650$ Ohms judged to be sufficiently mature for monolayer imaging.

\section{Fluorescent Labeling of Plasma Membrane}

Lipid Dyes. Prior to imaging, CaCo2A monolayers, which were grown on 8-well slides, were treated with $2 \mu \mathrm{M}$ FM143 (553 nm, Abs: 570 nm, Em.) (Molecular Probes), diluted in Dulbecco's phosphate buffered saline from a stock solution, and incubated at $37^{\circ} \mathrm{C}, 5 \% \mathrm{CO}_{2}$ for 2 hours.

\section{Anti-Occludin Immunofluorescence}

CaCo2A cells were grown to confluence on collagencoated 18-mm glass coverslips in 6 well plates. Monolayers were incubated in ethanol for 30 minutes at $4^{\circ} \mathrm{C}$, followed by incubation with acetone stored at $-20^{\circ} \mathrm{C}$ for 3 minutes at room temperature. Samples were blocked (2\% Natural Goat Serum, 2\% BSA, 0.2\% Dehydrated Milk, 25 mls DPBS) for 1.5 hours, incubated inverted on parafilm with $2 \mu \mathrm{g} / \mathrm{ml}$ monoclonal mouse anti-human occludin antibody overnight at $4^{\circ} \mathrm{C}$ followed by incubation with $10 \mu \mathrm{g} / \mathrm{ml}$ Texas Red labeled goat anti-mouse antibody for 1 hour at room temperature.

\section{Shiga Toxin Purification, Labeling, and Detection}

Purified Shiga toxins were obtained from the New England Medical Center (Boston, MA) GRASP core facility, as previously described (Hurley et al., 1999). The toxins were diluted to $1 \mathrm{mg} / \mathrm{mL}$ in $1 \mathrm{M}$ sodium bicarbonate, $\mathrm{pH} 7.2$ in solution. Proteins were directly labeled with Oregon Green (Molecular Probes), according to the manufacturer's protocol, using $20 \mathrm{mg}$ dye stock per sample. SDS-PAGE (15\% separating gels) were loaded with $5 \mathrm{mg}$ of labeled and unlabeled toxins and compared with low molecular weight standards following Coomassie blue staining. Gels were analyzed under ultraviolet light to verify the presence of fluorescent labeled toxins. Toxins were incubated with live CaCo2A monolayers at $37^{\circ}$ for 1-2 hours, and cells were subsequently imaged by two-photon microscopy.

\section{Two-Photon Excitation Microscopy}

Multiphoton microscopy offers several advantages for deep tissue imaging compared to more conventional microscopy techniques, such as confocal microscopy. Through use of the two-photon effect, a quantum mechanical phenomenon, the simultaneous absorption of two longer wavelength photons, can induce electronic transitions (Fig. 1). The use of less energetic photons offers two principal advantages, from the imaging perspective. The first advantage is reduced photo-damage since photo-toxicity effects are roughly inversely proportional to wavelength. The second advantage results from the increased penetration depth of longer wavelength light due to reduced Rayleigh scattering and tissue absorption. An important property of multiphoton microscopy takes advantage of the quadratic dependence of two-photon absorption on cross-section excitation intensity. Fluorescence mainly occurs in re- gions of incident photon flux. Therefore, efficient twophoton excitation occurs only near the image focal plane leading to excellent three-dimensional resolution. In this manner, multi-photon excitation can be limited to a highly discrete excitation volume, which can be systematically varied scanned, across an $x-y$ plane, and through multiple $\mathrm{z}$ planes.

Two-photon excitation microscopy (excitation wavelength $780 \mathrm{~nm}$ ) was used to image epithelial monolayer cultures in vivo. A schematic diagram of the experimental preparation is shown in Figure 2. Tissue specimens were imaged with a mode-locked Ti-sapphire laser, with a $76-\mathrm{MHz}$ repetition rate and a 150 -fsec pulse width (Coherent, Inc, Palo Alto, CA). The power of the laser light source was regulated with a GlanThompson polarizer, which, given a transmission efficiency of $10-20 \%$, resulted in approximately $15 \mathrm{~mW}$ at the level of the monolayer of cells. The beam expanded laser light was directed into the microscope via a galvanometer-driven x-y scanner (Cambridge Technology, Watertown, MA), and images were generated by raster scanning the $\mathrm{x}-\mathrm{y}$ mirrors. The excitation light entered the microscope via a modified epi-fluorescence light pathway, while the scan lens was positioned such that the $x-y$ scanner was at its eye-point while the field aperture was at its focal point. The axial position of the objective was driven by a piezoelectric driver with a resolution of $0.05 \mu \mathrm{m}$ over $100 \mu \mathrm{m}$. The typical frame rate (per single slice image) was approximately 3 seconds.

In order to maximize the number of available photons for detection (while minimizing potential photodamage), we used a single-photon counting system in which the fluorescence signal at each pixel was detected by an R5600-P photo-multiplier tube (Hamamatsu, Bridewater, NJ). This constitutes a photon counting module with high quantum efficiency ( $12 \%$ at $500 \mathrm{~nm}$ and $25 \%$ at $400 \mathrm{~nm}$ ). A $100 \mathrm{MHz}$ single-photon counting discriminator (F100T, Advanced Research Instruments, Boulder, CO) converted single-photon bursts into TTL pulses, which were subsequently counted and saved on a PC computer (Gateway) as photon intensity modulated images. The 3D deep-tissue volume scans produced $256 \times 256$ images with a resolution $1.85 \times 1.85 \mu \mathrm{m}$ and a slice thickness of $5 \mu \mathrm{m}$. The maximum achievable scan depth was approximately $100 \mu \mathrm{m}$.

\section{RESULTS}

In order to display the structural relationships between contiguous $\mathrm{CaCo} 2 \mathrm{~A}$ mature epithelial cells in monolayer culture, we have shown a three-dimensional image of these cells in the intact monolayer, in which the plasma membranes were labeled in vivo by a highly lipophilic probe. The maturity of the constituting cells of the monolayer was suggested by the length of the growth period, and confirmed by the presence of a high electrical resistance. As shown in Figure 3, the cells, viewed from the superior and lateral surfaces, display a highly regular hexagonal configuration. When viewed as a cross-section through the monolayer, the cells display the configuration of a series of elongated cylinders. We have imaged these cells in the monolayer alternatively with a label, which identifies proteins 


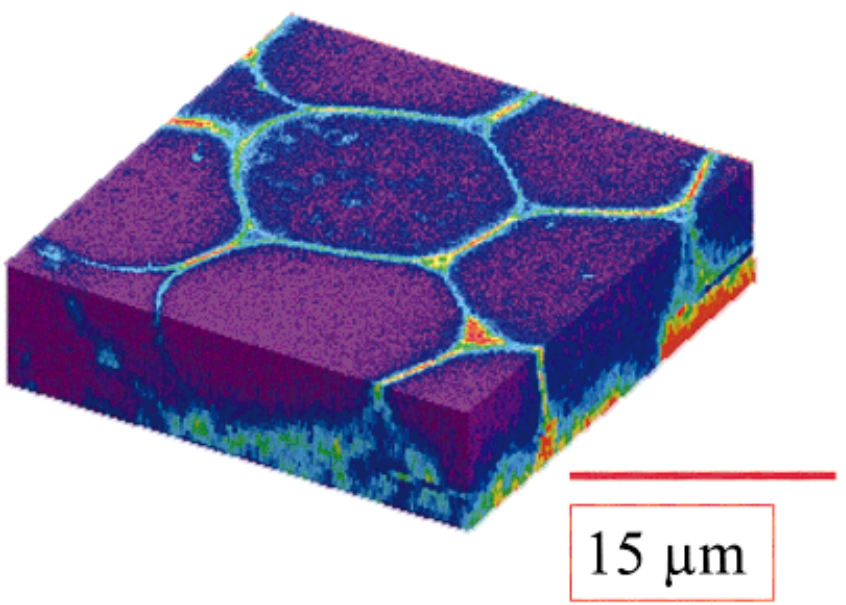

Fig. 3. 3D cytoarchitecture of $\mathrm{CaCo} 2 \mathrm{~A}$ cells in monolayer culture: Labeling of the plasma membrane. Cell membranes for CaCo2A cells grown to confluency were labeled with FM143 dye at $37^{\circ} \mathrm{C}$ for 2 hours, and viewed under two-photon microscopy, as described. Shown is a 3D reconstruction of a stacked series of $2 \mathrm{D}$ images. Cells, when viewed from the superior and lateral directions, displayed a highly homogeneous pattern of interconnected hexagonal cylinders. Scale bar $=$ $10 \mu \mathrm{m}$.

associated with the intercellular tight junctions, namely zonule occudin, $\mathrm{ZO}$, and have displayed the labeled monolayer as a $3 \mathrm{D}$ data set. As shown in Figure 4 , the cells may be conceived as a series of adjacent cylindrical structures. In the case of both membrane labels, the majority of cells completely traverse the imaged tissue monolayer. Of particular note for the $\mathrm{ZO}$ stained cells, the label is found predominantly at the apical surface, but can be readily detected at all vertical levels of the culture monolayer.

One of the principal utilities for deep in situ fluorescence imaging is the ability to visualize spatially complex biological functions. In Figure 5, we have illustrated one such phenomenon, that of the attachment and internalization of the enteric pathogen, Shiga toxin. This toxin is produced by an enteropathogenic Escherichia coli, which is known to produce both mucosal inflammatory disease, in the form of bloody diarrhea, and systemic disease, in the form of hemolyticuremic syndrome. We have shown here an example of a stacked series of 2D images depicting the internalization of Shiga toxin, following in vivo incubation with live cells. Note that distinct variations exist in the extent of toxin internalization as a function of the depth of microscopic imaging. In all cases, it is possible to discern that initial toxin localization occurs at or adjacent to the plasma membrane specifically at points of intercellular attachment to each other and presumably in association with a binding event. This is followed by step-wise internalization into the cytosol, whose net effect is to distribute the toxin laterally from the perspective of the cell surface, and vertically through the monolayer to accomplish translocation of the toxin.

\section{DISCUSSION}

The key functions of the mucosal layer of the GI tract include the binding and internalization of nutrients,

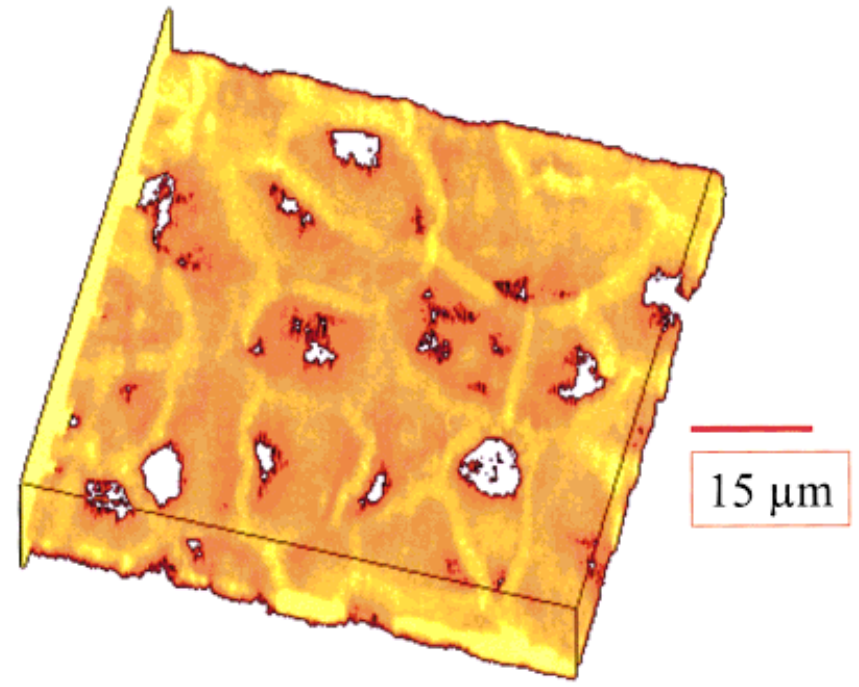

Fig. 4. 3D cytoarchitecture of $\mathrm{CaCo} 2 \mathrm{~A}$ cells in monolayer culture: labeling of intercellular tight junctions. CaCo2A cells were labeled for zonule occludin ( $\mathrm{ZO}$ ), as described in the text. Shown is a $3 \mathrm{D}$ reconstruction of a stacked series of $2 \mathrm{D}$ images. The edges of the rectangular shape comprising the monolayer are outlined in black. On the left of the image is depicted a yellow vertically-oriented plane in order to enhance $3 \mathrm{D}$ perception of the image. Viewed with $\mathrm{ZO}$ labeling, the cell membranes are conceived as a series of adjacent and connected cylinders, which in most cases traverse the entire monolayer (shown as a white signal void). Scale bar $=10 \mu \mathrm{m}$.

absorption and secretion of fluids and proteins, and the regulation of the gut-pathogen relationships. Since mature mucosal cells of the GI tract are characteristically polarized in situ, it is reasonable to presume that many aspects of their function are related to the ability of these cells to distribute substances in a geometrically specific manner to either the apical or basal portions of the cells. The physiological mechanisms by which these cells translocate proteins and other substances can best be conceived in a setting in which intracellular molecules can be labeled and tracked in vivo. However, obtaining the deep tissue and cell imaging simultaneous with assaying tissue physiology has been elusive for conventional microscopists, owing to the inability of conventional microscopy to penetrate deeply without light scattering and the induction of cytological damage.

Two-photon excitation microscopy may constitute a superior microscopic method to conventional microscopy for attaining these goals. The central technology underlying two-photon imaging is the use of ultrafast laser excitation, with the ability to excite multiple photons at discrete femtoliter volumes of tissue (Denk et al., 1990). The tissue can be sequentially sampled across a slice and through multiple slices to achieve a complete $3 \mathrm{D}$ volume, without significant adverse effects on the underlying biology of the tissue or cell. The use of comparable multi-slice imaging of cells or tissue using confocal microscopy presents several technical problems, which has greatly limited its use in physiological imaging (Centonze and White, 1998). These problems include the high degree of light scattering 
A

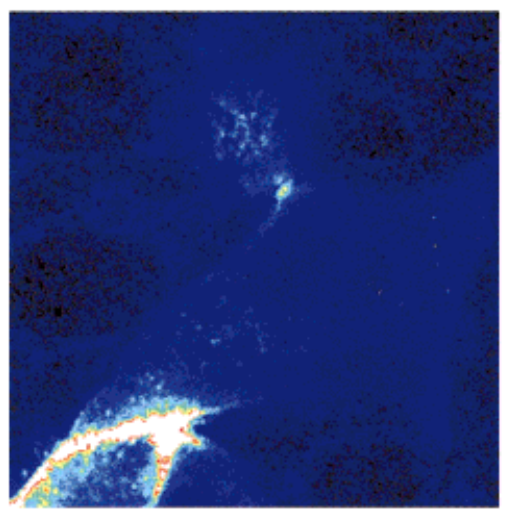

$\mathrm{C}$

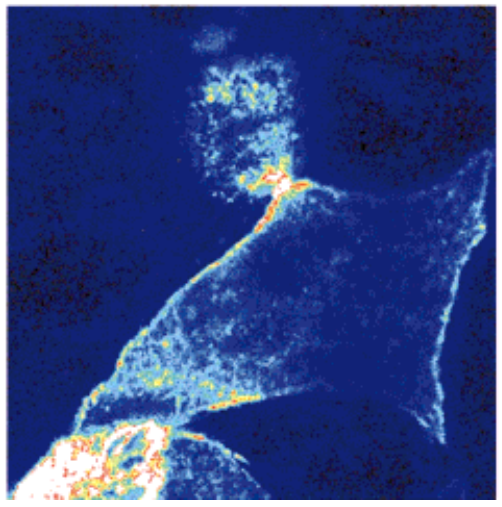

B

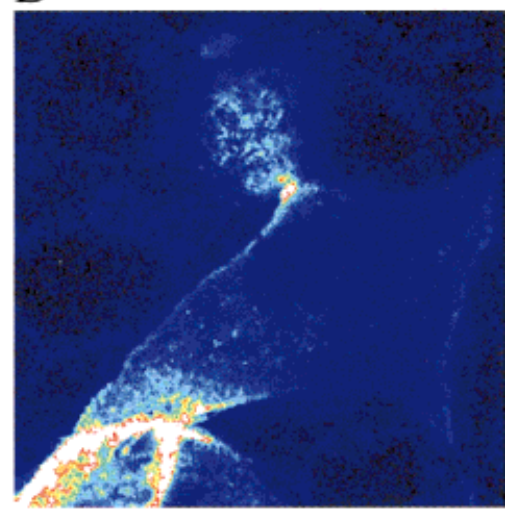

$\mathrm{D}$

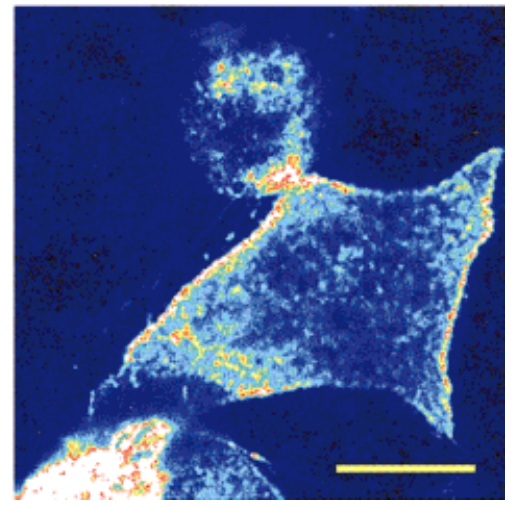

Fig. 5. 3D distribution of incorporated Shige toxin within CaCo2A cells. Purified Shige toxins were labeled with Oregon Green, and analyzed under UV light to confirm effectiveness of labeling. Toxins were incubated at $37^{\circ} \mathrm{C}$ with $\mathrm{CaCo} 2 \mathrm{~A}$ monolayers, and imaged with two-photon microscopy. Shown is a series of $2 \mathrm{D}$ images depicting the differences of toxin distribution as a function of $\mathrm{z}$ position following translocation into the cytosol. Slice thickness xxx microns, and inter-slice interval xxx microns. Of note, initial toxin localization tended to occur at points in the plasma membrane adjacent to intercellular attachments, followed by a step-wise internalization into the cytosol. Scale bar $=$ $10 \mu \mathrm{m}$. that occurs with increasing depth of imaging, leading to significant image degradation, a high degree of light scattering owing to the fact that the extent of scattering is proportional to the wavelength of the excitation light (i.e., shorter wavelengths result in higher scattering, and longer wavelengths results in lower scattering), the common occurrence of photo-bleaching of the fluorophore outside of the focal plane, and the need for a blocking pinhole to achieve optical sectioning. In contrast, fluorophore excitation with two-photon microscopy is confined to the volume of interest, and therefore there is no need for a blocking pinhole and all the signal generated by the sample can be collected by the detector, i.e., higher signal to noise. The particular applicability of two-photon microscopy for depth discrimination in thick tissues results from the fact that fluorescence signal intensity is highly dependent on the degree of photon flux, an attribute that decreases rapidly as a function of distance from the focal plane (Fig. 6).

As GI tract epithelial cells mature in culture, cell structure begins to approximate that of the intestinal villus cell. These cells, in particular, manifest an elongated and cylindrical geometry, distinct apical-to-basal polarity, an apical brush border, and tight junctions (Madara, 1998; Madara et al., 1987; Quaroni and Beaulieu, 1997; Peterson and Mooseker, 1993). The latter are a highly specialized convergence of membrane proteins, cytoskeletal fibers, and signalling molecules, re- sponsible for regulating the transcellular flux of solutes. Due to the capacity of these culture monolayers to simulate normal intestinal function, they have been extensively employed to study the mechanisms of pathogen attachment, internalization, and intoxication, drug actions and interactions, nutrient translocation, and the complex array of molecular mediators that affect tissue inflammatory responses. However, these studies are limited in that the study of structure cannot be done concurrently with the study of function. The ability to image both structure and function synchronously in live cells should open new and creative methodological directions for the study of GI mucosal pathobiology.

We have provided, in this initial report, a description of the application of two-photon microscopy to imaging to the gut epithelium, as well as offering several novel observations regarding its structure and function. In monolayer culture, once confluent, the $\mathrm{CaCo} 2 \mathrm{~A}$ cells align themselves as regular parallel cylinders, approximately hexagonal at the luminal surface, similar in a sense to a row of beer cans. This pattern can be readily discerned independent of the method of labelling the membrane. In the present study, we used FM dyes as a strongly lipophilic membrane label, and antibody directed to the tight junction protein, zonule occludens. We additionally demonstrated the ability to track internalized substances, in this Shiga toxin binding subunit, across 


\section{Two-Photon}
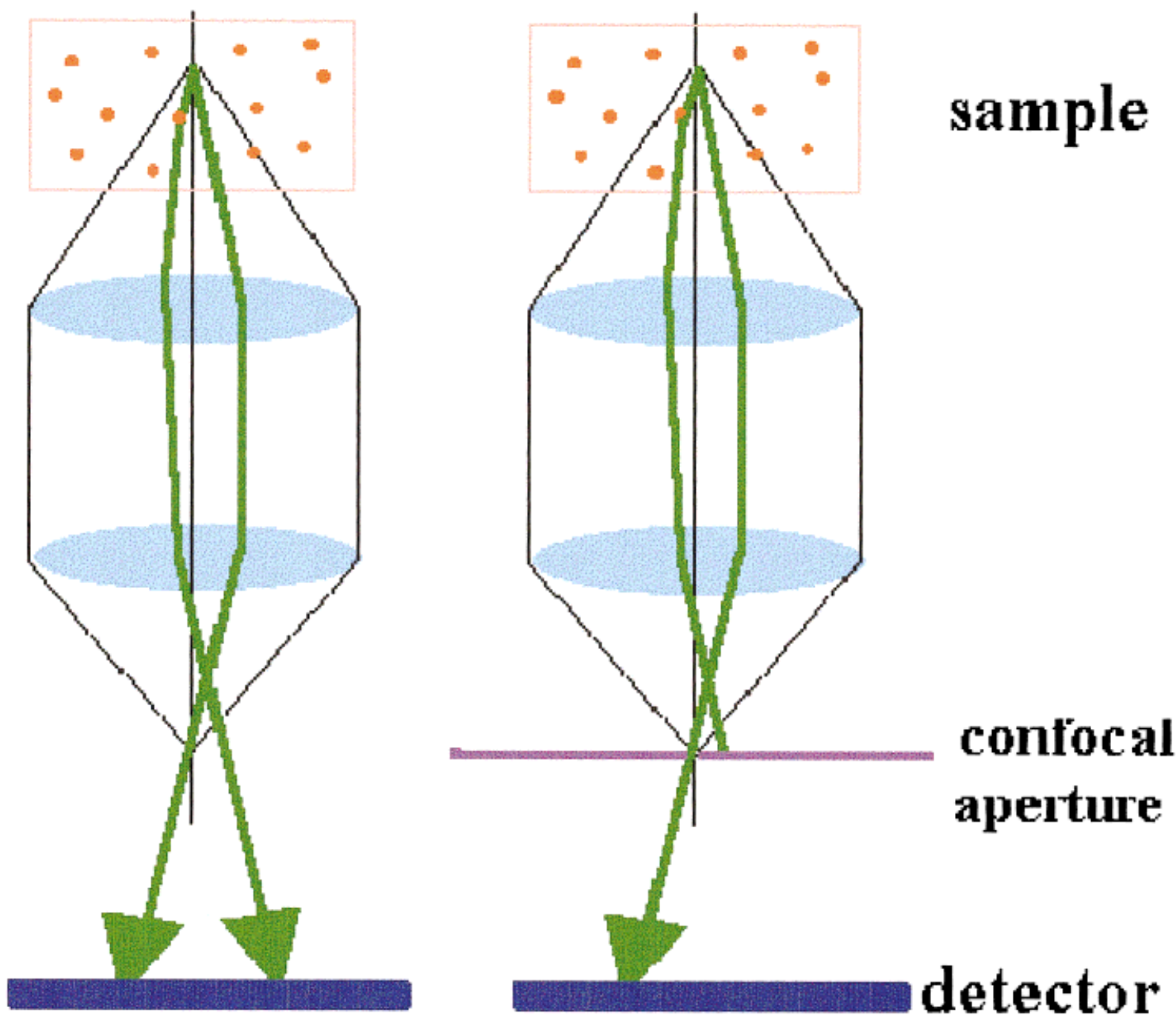

Fig. 6. Comparison between two-photon and confocal microscopy. The principal advantage of two-photon microscopy over conventional confocal microscopy for 3D imaging in tissue is based upon the fact that two-photon excitation is confined to a discrete volume of interest, thus eliminating the need for a blocking pinhole (confocal), and allowing more efficient signal collection. Shown is the photon collection paradigm for two-photon microscopy (left) and conventional confocal

the lateral surface and through multiple layers of the cells, thus providing a depiction of 3D distribution of the internalized toxin.

We conclude that two-photon excitation microscopy is a viable method for achieving three-dimensional structural and functional imaging of gut epithelial cells in culture, without imposing significant tissue and cell damage secondary to laser excitation. Future research applications in the area of gut mucosal biology using two-photon microscopy will likely include discerning the mechanisms of pathogen binding, uptake, and toxicity, dissecting the cellular machinery associated with protein translocation and excretion, and better understanding the molecular basis of nutrient absorption. In
Confocal

microscopy (right). Two-photon advantages over confocal microscopy also include diminished radiation loss due to light scatter and absorption, since longer excitation wavelengths are used, and minimal tissue or cell damage due to photo-excitation, since the volume of sample that actually interacts with the excitation light is small per unit time. (note color version of artwork is available online.)

addition, live cell imaging with two-photon microscopy may play an important role in drug discovery, since multiple compounds can be successfully screened and biological responses assayed.

\section{REFERENCES}

Centonze VE, White JG. 1998. Multiphoton excitation provides optical sections from deeper within scattering specimens than confocal imaging. Biophys J 75:2015-2024.

Denk W, Strickler JH, Webb WW. 1990. Two-photon laser scanning fluorescence microscopy. Science 248:73-76.

Hurley BP, Jacewicz M, Thorpe CV, Lincicome LL, King AJ, Keusch GT, Acheson DWK. 1999. Shiga toxins 1 and 2 translocate differently across polarized intestinal epithelial cells. Infection Immunity 67:6670-6677. 
Madara JL. 1990. Pathobiology of the intestinal epithelial barrier. Am J Pathol 137:1273-1281.

Madara JL. 1998. Regulation of the movement of solutes across tight junctions. Annu Rev Physiol 60:143-159.

Madara JL, Stafford J, Dharmsathaphorn K, Carlson S. 1987. Structural analysis of human intestinal epithelial cell line. Gastroenterology 92: $1133-1145$.

Masters B, So PTC, Gratton E. 1997. Multiphoton excitation fluorescence microscopy and spectroscopy of in vivo human skin. Biophys J 72:2405-22412.

McCormick BA, Colgan SP, Archer CD, Miller SI, Madara JL. 1993. Salmonella typhymurium attachment to human intestinal epithelial monolayers: transcellular signalling to subepithelial neutrophils. JCB 123:895-907.

Michalsky MP, Deitch EA, Ding J, Lu Q, Huang Q. 1997. Interleukin 6 and tumor necrosis factor production in an enterocyte cell model (Caco-2) during exposure to Escherichia coli. Shock 2:139146.
Nusrat A, Giry M, Turner JR, Colgan SP, Parkos CA, Carnes D, Lemichez E, Boquet P, Madara JL. 1995. Rho protein regulates tight junctions and perijunctional actin organization in polarized epithelia. PNAS 92:10629-1063.

Perdikis DA, Basson MD. 1997. Basal nutrition promotes human intestinal epithelial (Caco-2) proliferation, brush border enzyme activity, and motility. Crit Care Med 25:159-165.

Peterson MD, Mooseker MS. 1993. An in vitro model for the analysis of intestinal brush border assembly. I Ultrastructural analysis of cell contact-induced brush border assembly in Caco-2Bbe cells. J Cell Sci 105:445-460.

Quaroni A, Beaulieu JF. 1997. Cell dynamics and differentiation of conditionally immortalized human intestinal epithelial cells. Gastroenterology 13:1198-1213.

Wick MJ, Madara JL, Fields BN, Normark SJ. 1991. Molecular cross talk between epithelial cells and pathogenic micro-organisms. Cell 67:651-659. 